\title{
Ptychographic Single Particle Analysis for Biological Science
}

Xudong Pei ${ }^{1}$, Liqi Zhou ${ }^{1}$, Judy kim ${ }^{2}$, Mark Boyce ${ }^{3}$, Huang Huang ${ }^{4}$, Emanuela Liberti ${ }^{5}$, Peter Nellist ${ }^{2}$, Peijun Zhang ${ }^{6}$, David Stuart ${ }^{7}$, Angus Kirkland ${ }^{8}$ and Peng Wang ${ }^{1}$

${ }^{1}$ Nanjing University, China (People's Republic), ${ }^{2}$ University of Oxford, Department of Materials, United States, ${ }^{3}$ Division of Structural Biology, Wellcome Trust Centre for Human Genetics, University Of Oxford, United Kingdom, ${ }^{4}$ The Rosalind Franklin Institute, Harwell Campus, United Kingdom, ${ }^{5}$ he Rosalind Franklin Institute, Harwell Campus, United Kingdom, ${ }^{6}$ Division of Structural Biology, Wellcome Trust Centre for Human Genetics, University of Oxford, Oxford, England, United States, ${ }^{7}$ Division of Structural Biology, Wellcome Trust Centre for Human Genetics, University of Oxford, United States, ${ }^{8}$ Department of Materials, University of Oxford, United Kingdom

Cryo-electron microscopy (cryo-EM), has attracted significant efforts to develop techniques for the high resolution three-dimensional structure characterization of biological samples in a close-to-native state at high resolution, including single particle analysis[1] and tilted tomography[2]. However, unstained biological samples embedded in thin vitreous ice are essentially pure phase objects that are extremely radiation sensitive[3], and consequently images of these have low signal-to-noise ratios[4] and low contrast[5,6]. To counter the latter, cryo-EM relies on the use of phase contrast imaging at high defocus to improve information transfer at low spatial frequencies at the expense of higher spatial frequencies[7]. Therefore, it is important to develop ways to improve resolution and contrast without damaging samples, including the use of direct electron detectors and novel phase plates[6]. However, the routine applications of phase plates are still somewhat restricted by signal attenuation at high frequencies, inconsistent fabrication, poor reliability and short working lifetimes due to electrostatic charging $[8,9]$.

An alternate technique is electron ptychography as shown in Fig. 1, where a probe, defocused by several tens of nm's is scanned over a specimen with highly overlapping probe positions. This approach has demonstrated its potential for super-resolution imaging[10,11], high-contrast light-element detection[12], low dose imaging[13] and three-dimensional imaging[14,15]. The development of advanced direct electron detectors dramatically increases the signal-to-noise ratio and improves the dose efficiency of ptychography[13] making it applicable to low dose biological imaging with high contrast and signal-to-noise ratios[16].

Here, we experimentally demonstrated low-dose ptychography with a defocused probe for biological imaging in a cryo-state in a scanning electron diffraction mode as shown schematically in Fig. 1(a and b) using a JEOL ARM300CF. Ptychographic diffraction datasets in Fig. 1(c).were acquired on a direct electron detector with the probe rastered across the samples. Rotavirus double-layered particles (DLPs) with a diameter of $76.5 \mathrm{~nm}$ were used as a typical example for the demonstration of this method under cryo conditions. The ptychographic phase of the rotavirus DLPs was reconstructed at a dose of $23 \mathrm{e} / \mathrm{A}^{2}$ as shown in Fig. 1 (d). At this dose, the phase image shows a strong contrast in the virus particles, where both the capsids of viral protein (VP) and channels between these can be clearly seen. These features are consistent with those observed using conventional defocused TEM images[Fig. 2 (a)]. The ptychographic phase preserves low spatial frequency information, facilitating the recovery of these important molecular features. Importantly by altering the probe convergence angle the ptychographic transfer function can be "tuned" to recover specific frequency ranges including better transfer of both low and high spatial frequencies when compared to conventional phase contrast imaging. We will show that it can be applied to the micron sized fields of view that are required for either imaging many indentical particles for single particle analysis[Fig. 2 (b)] or capturing multiple biological 
ultrastructures in a cellular context. Finally, we will show that ptychographic reconstruction can also be readily combined with single particle analysis and tomography to yield 3D reconstructions of biological samples.

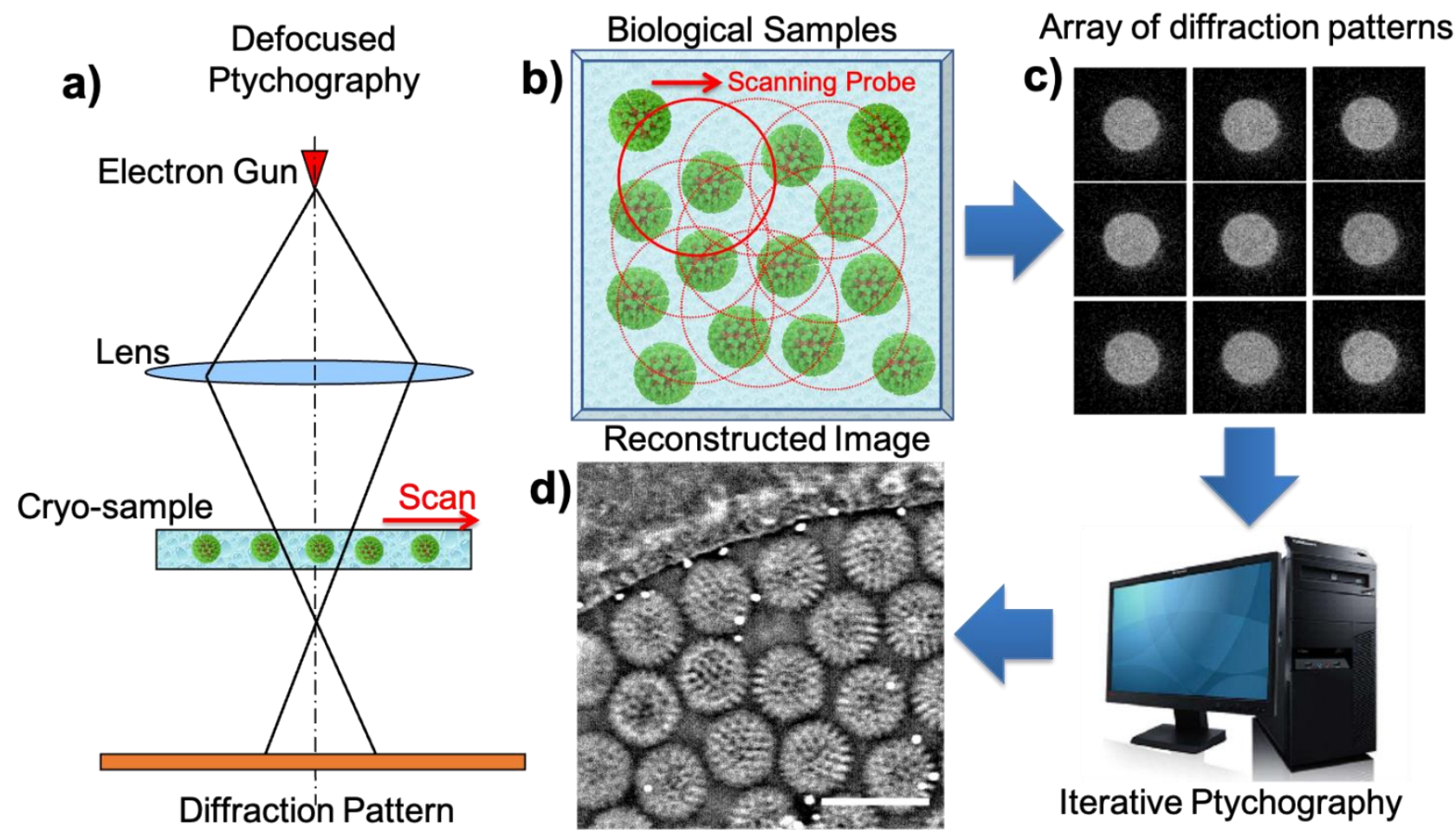

Figure 1. Schematic diagram of workflow used for cryo-ptychography. (a) Optical configuration; (b) Schematic biological samples at a cryo-state; (c) Array of diffraction patterns as a function of probe positions; (d) Reconstructed phase of rotavirus double-layered particles. Scale bars: $100 \mathrm{~nm}$

a)

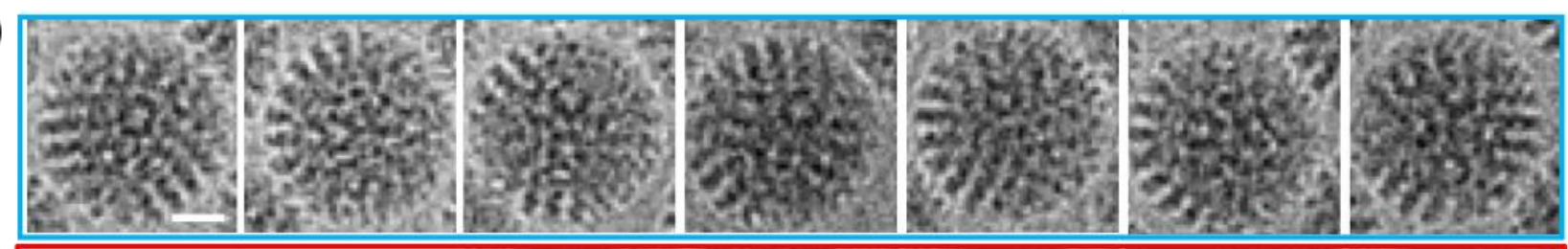

b)

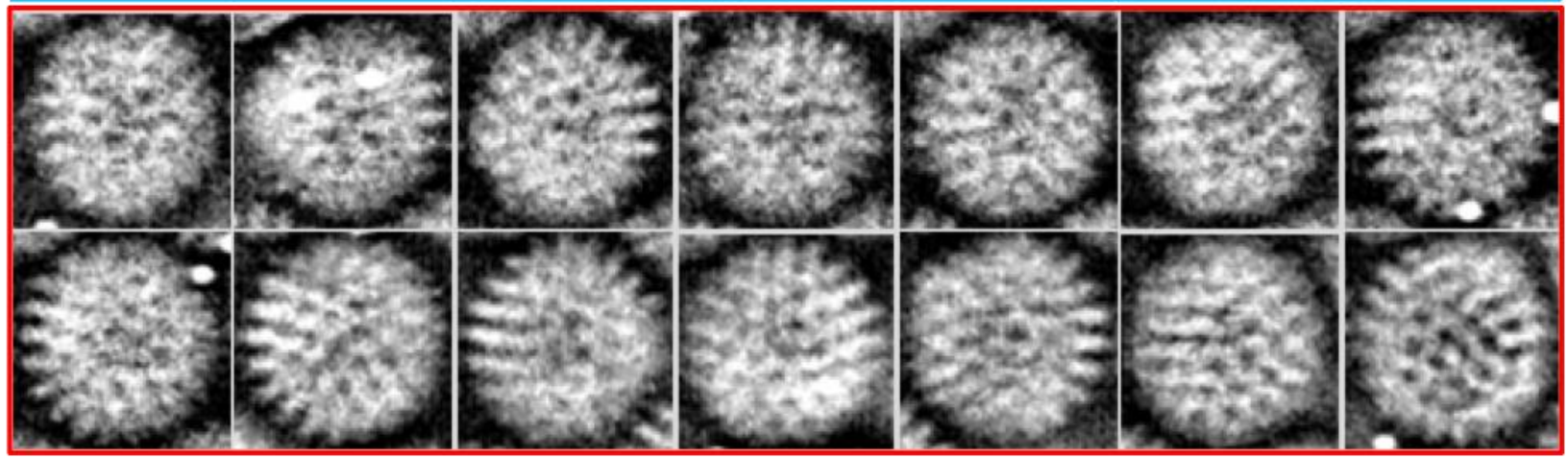

Figure 2. Many instances of the viral particles for single particle analysis can be extracted from (a) TEM images and (b) reconstructed ptychographic phases. Scale bars: $20 \mathrm{~nm}$

References 
1] R. Henderson et al., Journal of Molecular Biology 213, 899 (1990).

[2] Lučić V, et al., The Journal of Cell Biology 202, 407 (2013).

[3] K. A. Taylor et al., Science 186, 1036 (1974).

[4] R. Henderson, Quarterly Reviews of Biophysics 28, 171 (2009).

[5] R. Danev et al., Ultramicroscopy 88, 243 (2001).

[6] R. Danev et al., Proceedings of the National Academy of Sciences 111, 15635 (2014).

[7] K. H. Downing et al., Ultramicroscopy 108, 921 (2008).

[8] R. Danev et al., Ultramicroscopy 109, 312 (2009).

[9] R. Danev et al., Current opinion in structural biology 46, 87 (2017).

[10] P. D. Nellist et al., Nature 374, 630 (1995).

[11] Y. Jiang et al., Nature 559, 343 (2018).

[12] P. Wang et al., Scientific Reports 7, 2857 (2017).

[13] J. Song et al., Scientific Reports 9, 3919 (2019).

[14] A. M. Maiden et al., J. Opt. Soc. Am. A 29, 1606 (2012).

[15] S. Gao et al., Nature Communications 8, 163 (2017).

[16] L. Zhou, et al., Nature Communications 11, 2773 (2020).

[17] The authors acknowledge funding from the National Natural Science Foundation of China (11874199). 